Pacific Journal of Mathematic 


\title{
ADDITION THEOREMS FOR SETS OF INTEGERS
}

\author{
Calvin T. Long
}

Let $C$ be a set of integers. Two subsets $A$ and $B$ of $C$ are said to be complementing subsets of $C$ in case every $c \in C$ is uniquely represented in the sum

$$
C=A+B=\{x \mid x=a+b, a \in A, b \in B\} .
$$

In this paper we characterize all pairs $A, B$ of complementing subsets of

$$
N_{n}=\{0,1, \cdots, n-1\}
$$

for every positive integer $n$ and show some interesting connections between these pairs and pairs of complementing subsets of the set $N$ of all nonnegative integers and the set $I$ of all integers. We also show that the number $C(n)$ of complementing subsets of $N_{n}$ is the same as the number of ordered nontrivial factorizations of $n$ and that

$$
2 C(n)=\sum_{d \mid n} C(d) .
$$

The structure of complementing pairs $A$ and $B$ has been studied by de Bruijn [1], [2], [3] for the cases $C=I$ and $C=N$ and by A. M. Vaidya [7] who reproduced a fundamental result of de Bruijn for the latter case. In case $C=N$ it is easy to see that $A \cap B=\{0\}$ and that $1 \in A \cup B$. Moreover, if we agree that $1 \in A$, it follows from the work of de Bruijn, that, except in the trivial case $A=N, B=\{0\}$, $A$ and $B$ are infinite complementing subsets of $N$ if and only if there exists an infinite sequence of integers $\left\{m_{i}\right\}_{i \geqq 1}$ with $m_{i} \geqq 2$ for all $i$, such that $A$ and $B$ are the sets of all finite sums of the form

$$
\begin{aligned}
a & =\sum x_{2 i} M_{2 i}, \\
b & =\sum x_{2 i+1} M_{2 i+1}
\end{aligned}
$$

respectively where $0 \leqq x_{i}<m_{i+1}$ for $i \geqq 0$ and where $M_{0}=1$ and $M_{i}=\Pi_{j=1}^{i} m_{j}$ for $i \geqq 1$. In the remaining case, when just one of $A$ and $B$ is infinite, the same result holds except that the sequence $\left\{m_{i}\right\}$ is of finite length $r$ and that $x_{r} \geqq 0$. Similar results can also be obtained in the case of complementing $k$-tuples of subsets of $N$ for $k>2$.

The case $C=I$ is much more difficult and, while sufficient conditions are easily given, necessary and sufficient conditions that a pair $A, B$ be complementing subsets of $I$ are not known. As an example of sufficient conditions, we note that if $A$ and $B$ are as in (1) above, then $A$ and $-B$ form a pair of complementing subsets of $I$. This is 
an immediate consequence of the fact that every integer $n$ can be represented uniquely in the form

$$
n=\sum_{i=0}^{r}(-1)^{i} x_{i} M_{i}
$$

with $x_{i}$ and $M_{i}$ as in (1). Incidentally, if $B$ is finite, it is not difficult to see that there exists an integer $r_{0} \leqq 0$ such that $A$ and $-B$ form a pair of complementing subsets of the set

$$
R=\left\{r \mid r \in I, r \geqq r_{0}\right\} .
$$

And if $A$ is finite, there exists an integer $s_{0}>0$ such that $A$ and $-B$ are complementing subsets of the set

$$
S=\left\{s \mid s \in I, s \leqq s_{0}\right\} .
$$

2. Complementing sets of order $n$. We now investigate the structure of pairs $A, B$ of complementing subsets of the set

$$
N_{n}=\{0,1, \cdots, n-1\}
$$

for integral values of $n \geqq 1$. Such a pair of sets will be called complementing sets of order $n$ and we will write $(A, B) \sim N_{n}$.

In case $n=1$, we have only the trivial pair $A=B=\{0\}$. For $n>1$, it is easy to see that $A \cap B=\{0\}$ and that $1 \in A \cup B$. We choose our notation so that $1 \in A$ and, if $m$ is the least positive element in $B$, then we also have that $N_{m} \subset A$ and that none of $m+1, m+2$, $\cdots, 2 m-1$ appear in either $A$ or $B$. If $B$ does not contain positive elements, we have only the trivial pair $A=N_{n}, B=\{0\}$.

For the remainder of the paper, we restrict our attention to the case $n>1$ and we use the notation $m S$ to denote the set of all multiples of elements of a set $S$ by an integer $m$.

Lemma 1. Let $A, B, C$, and $D$ be subsets of $N_{n}$ such that, for $a$ fixed integer $m \geqq 2$,

$$
A=m C+N_{m} \quad \text { and } \quad B=m D .
$$

Then $(A, B) \sim N_{m p}$ if and only if $(C, D) \sim N_{p}$ where $p \geqq 1$.

Proof. Suppose first that $(C, D) \sim N_{p}$. Then, for any $s \in N_{m p}$, there exist integers $q \in N_{p}$ and $r \in N_{m}$ such that $s=m q+r$. Since $(C, D) \sim N_{p}$, there exist $c \in C$ and $d \in D$ such that $q=c+d$. But then

$$
s=m(c+d)+r=(m c+r)+m d=a+b
$$

with $a=m c+r \in A$ and $b=m d \in B$. Moreover, if this representation 
is not unique, there exist $a^{\prime} \in A, b^{\prime} \in B, c^{\prime} \in C, d^{\prime} \in D$, and $r^{\prime} \in N_{m}$ such that

$$
s=a^{\prime}+b^{\prime}=\left(m c^{\prime}+r^{\prime}\right)+m d^{\prime} .
$$

But then $r=r^{\prime}$ and

$$
c+d=q=c^{\prime}+d^{\prime}
$$

and this violates the condition that $q$ be uniquely represented in the sum $C+D$.

Conversely, suppose that $(A, B) \sim N_{m p}$. Then, for $s \in N_{p}$, there exist $a \in A, b \in B, c \in C, d \in D$, and $r \in N_{m}$ such that

$$
s m=a+b=(m c+r)+m d .
$$

But this implies that $r=0$ and that $s=c+d$. Also, if this representation of $s$ in $C+D$ is not unique, there exist $c^{\prime} \in C$ and $d^{\prime} \in D$ such that $s=c^{\prime}+d^{\prime}$. But then

$$
s m=c m+d m=c^{\prime} m+d^{\prime} m
$$

and this violates the condition that $s m$ be uniquely represented in $A+B$.

The next lemma is an adaptation of a key result of de Bruijn [2, p. 16].

Lemma 2. If $(A, B) \sim N_{n}$, then there exist an integer $m \geqq 2$ such that $m \mid n$ and a complementing pair $A^{\prime}, B^{\prime}$ of order $n / m$, with $1 \in A^{\prime}$ if $B \neq\{0\}$, such that

$$
A=m B^{\prime}+N_{m} \quad \text { and } \quad B=m A^{\prime} .
$$

Proof. If $B=\{0\}$, then $A=N_{n}$ and the desired result follows with $A^{\prime}=B^{\prime}=\{0\}$ and $m=n$. If $B \neq\{0\}$, let $m$ be the least positive integer in $B$. Since $1 \in A$ and $A \cap B=\{0\}$, it follows that $m \geqq 2$. Determine the integer $h$ such that

$$
h m \leqq n<(h+1) m .
$$

Now the induction of de Bruijn's proof holds for all nonnegative integers less than $h$ and shows that all elements of $B$ less than $h m$ are multiples of $m$ and that, for each $k$ with $0 \leqq k \leqq h-1$, the set

$$
\{k m, k m+1, \cdots, k m+m-1\}
$$

is either a subset of $A$ or is disjoint from $A$. This implies that $A^{\prime}$ and $B^{\prime}$ exist such that (1) holds and $1 \in A^{\prime}$ provided we are able to show that $h m+r \notin A \cup B$ for every integer $r \geqq 0$. Contrariwise, 
suppose that $h m+r \in A$. Then $h m+r+m \in A+B=N_{n}$, and this is impossible since $h m+r+m \geqq h m+m>n$. Similarly, if $h m+r \in B$, then $(m-1)+h m+r \in A+B$ and we have the same contradiction. Thus (3) holds and it follows that $m$ divides $n$ and, by Lemma 1 , that $\left(A^{\prime}, B^{\prime}\right) \sim N_{n / m}$.

The following theorem, which characterizes all complementing pairs of order $n>1$, now follows by repeated application of Lemma 2 .

THEOREM 1. Sets $A_{1}$ and $B_{1}$ form a complementing pair of order $n \geqq 2$ if and only if there exists a sequence $\left\{m_{i}\right\}_{i=1}^{r}$ of integers not less than two such that

$$
n=\sum_{i=1}^{r} m_{i}
$$

and such that $A_{1}$ and $B_{1}$ are the sets of all finite sums of the form

$$
a=\sum_{i=0}^{[(r-1) / 2]} x_{2 i} M_{2 i} \quad \text { and } \quad b=\sum_{i=0}^{[(r-2) / 2]} x_{2 i+1} M_{2 i+1}
$$

respectively with $M_{0}=1, M_{i+1}=\Pi_{j=1}^{i+1}=m_{j}$ and $0 \leqq x_{i}<m_{i+1}$ for $0 \leqq$ $i<r$. If $r=1$, we interpret the notation to mean that $B_{1}=\{0\}$.

It follows from Theorem 1 that there exists a one to one correspondence between the set $\mathscr{C}_{n}$ of all pairs of complementing sets of order $n>1$ and the set of all ordered finite sequences $\left\{m_{i}\right\}$ with $m_{i} \geqq 2$ such that $\Pi m_{i}=n$. Thus, if $C(n)$ denotes the number of elements of $\mathscr{C}_{n}$, then $C(n)$ is equal to the number $F(n)$ of ordered nontrivial factorizations of $n$. Curiously, as shown by P. A. MacMahon [4; p. 108], $F(n)$ is in turn equal to the number of perfect partitions of $n-1$. This last result is also listed by Riordan [6; pp. 123-4]. In a second paper, MacMahon [5; pp. 843-4] shows that

$$
C(n)=\sum_{j=1}^{q} \sum_{i=0}^{j-1}(-1)^{i}\left(\begin{array}{l}
j \\
i
\end{array}\right) \sum_{h=1}^{r}\left(\begin{array}{c}
\alpha_{h}+j-i-1 \\
\alpha_{h}
\end{array}\right)
$$

where $q=\sum_{h=1}^{r} \alpha_{h}$ and $n=\prod_{h=1}^{r} p_{h}^{\alpha}$ is the canonical representation of $n$. However, if one actually wants the values of $C(n)$, they are much more easily computed using the result of the following theorem:

THEOREM 2. If $n>1$ is an integer, then

$$
C(n)=\frac{1}{2} \sum_{d \mid n} C(d)=2 \sum_{d \mid n} \mu(d) C(n / d)
$$

where $\mu$ denotes the Möbius function. 
Proof. It follows from Lemma 2 that to each of the $C(n)$ distinct complementing pairs $A, B$ of order $n$ there corresponds a unique complementing pair $A^{\prime}, B^{\prime}$ of order $d$ where $d \mid n$ and $1 \leqq d<n$. Hence,

$$
C(n) \leqq \sum_{d \mid n, d<n} C(d) \text {. }
$$

Moreover, from each of the $C(d)$ distinct complementing pairs $C, D$ of order $d$, with $1 \leqq d<n$ and $1 \in D$ if $d \neq 1$, can be formed precisely one pair $A, B$ of complementing sets of order $d q=n$ by the method of Lemma 1. Since the new pairs formed in this way are clearly distinct, it follows that

$$
C(n) \geqq \sum_{d \mid n, d<n} C(d) .
$$

Thus, equality holds and this implies that

$$
C(n)=\frac{1}{2} \sum_{d \mid n} C(d)
$$

as claimed. The other equality is an immediate consequence of the Möbius inversion formula.

Except for Theorem 2, the preceding theorems reveal a striking parallel between the structure of complementing subsets of $N$ and the structure of complementing pairs of order $n$. The next theorem exhibits an additional interesting connecting between these two classes of pairs. Also, it is clear that a similar theorem holds giving sufficient conditions that $A$ and $B$ form a pair of complementing subsets of $I$.

THEOREM 3. Let $\left\{m_{i}\right\}_{i \geqq 1}$ and $\left\{M_{i}\right\}_{i \geq 0}$ be as defined in (1) above and let $\left(C_{i}, D_{i}\right) \sim N_{m_{i+1}}$ for $i \geqq 0$. If $A$ and $B$ are the sets of all finite sums of the form

$$
a=\sum c_{i} M_{i} \quad \text { and } \quad b=\sum d_{i} M_{i}
$$

respectively with $c_{i} \in C_{i}$ and $d_{i} \in D_{i}$ for $i \geqq 0$, then $(A, B) \sim N$.

Proof. Let $n$ be any nonnegative integer. Then $n$ can be represented uniquely in the form

$$
n=\sum_{i=0}^{r} e_{i} M_{i}
$$

with $e_{i} \in N_{m_{i+1}}$ for all $i$. Since $\left(C_{i}, D_{i}\right) \sim N_{m_{i+1}}$, there exist $c_{i} \in C_{i}$ and $d_{i} \in D_{i}$ such that $e_{i}=c_{i}+d_{i}$ uniquely. Therefore,

$$
\begin{aligned}
n & =\sum_{i=0}^{r}\left(c_{i}+d_{i}\right) M_{i} \\
& =\sum_{i=0}^{r} c_{i} M_{i}+\sum_{i=0}^{r} d_{i} M_{i} \\
& =a+b
\end{aligned}
$$


with $a \in A$ and $b \in B$. If this representation of $n$ in $A+B$ is not unique, there exist $a^{\prime} \in A$ and $b^{\prime} \in B$ such that

$$
n=a^{\prime}+b^{\prime}
$$

where

$$
a^{\prime}=\sum_{i=0}^{s} c_{i}^{\prime} M_{i} \quad \text { and } \quad b^{\prime}=\sum_{i=0}^{s} d_{i}^{\prime} M_{i}
$$

with $c_{i}^{\prime} \in C_{i}$ and $d_{i}^{\prime} \in D_{i}$ for each $i$. But then

$$
n=\sum_{i=0}^{s}\left(c_{i}^{\prime}+d_{i}^{\prime}\right) M_{i}
$$

and $c_{i}^{\prime}+d_{i}^{\prime} \in N_{m_{i+1}}$ since $\left(C_{i}, D_{i}\right) \sim N_{m_{i+1}}$ for all $i$. Since representations of $n$ in this form are unique, it follows that $r=s$ and that

$$
c_{i}+d_{i}=c_{i}^{\prime}+d_{i}^{\prime}
$$

for each $i$. And this violates the condition that $\left(C_{i}, D_{i}\right) \sim N_{m_{i+1}}$. Thus, the representation is unique and $(A, B) \sim N$ as claimed.

Note that if $r$ is fixed and $0 \leqq i<r$ in the sums defining $A$ and $B$ in the preceding theorem, then we conclude in the same way that $(A, B) \sim N_{n}$.

The author acknowledges his indebtedness to Professors Ivan Niven and E. A. Maier who made several helpful suggestions concerning the writing of this paper.

\section{REFERENCES}

1. N. G. de Bruijn, On bases for the set of integers, Publ. Math. (Debrecen) 1 (1950), 232-242.

2. - On number systems, Nieuw Arch. Wisk. 4 (1956), 15-17.

3. - Some direct decompositions of the set of integers, Math. Comp. 18 (1964), $537-546$.

4. P. A. MacMahon, The theory of perfect partitions and the compositions of multipartite numbers, Mess. Math. 20 (1891), 103-119.

5. - Memoir on the theory of compositions of numbers, Philos. Trans. Roy. Soc. London (A) 184 (1893), 835-901.

6. J. Riordan, An Introduction to Combinatorial Analysis, John Wiley and Sons, New York, 1958.

7. A. M. Vaidya, On complementing sets of non-negative integers, Math. Mag. 39 (1966), 43-44.

Received February 15, 1966, and in revised form August 22, 1966.

WAShington State University 


\section{PACIFIC JOURNAL OF MATHEMATICS}

\section{EDITORS}

\section{H. ROYDEN}

Stanford University

Stanford, California

J. P. JANS

University of Washington

Seattle, Washington 98105

\section{J. DUGUNDJI}

Department of Mathematics

Rice University

Houston, Texas 77001

RICHARD ARENS

University of California

Los Angeles, California 90024

ASSOCIATE EDITORS

E. F. BECKENBACH

B. H. NeumanN

F. WoLF

K. YosidA

\section{SUPPORTING INSTITUTIONS}

\author{
UNIVERSITY OF BRITISH COLUMBIA \\ CALIFORNIA INSTITUTE OF TECHNOLOGY \\ UNIVERSITY OF CALIFORNIA \\ MONTANA STATE UNIVERSITY \\ UNIVERSITY OF NEVADA \\ NEW MEXICO STATE UNIVERSITY \\ OREGON STATE UNIVERSITY \\ UNIVERSITY OF OREGON \\ OSAKA UNIVERSITY \\ UNIVERSITY OF SOUTHERN CALIFORNIA
}

\author{
STANFORD UNIVERSITY \\ UNIVERSITY OF TOKYO \\ UNIVERSITY OF UTAH \\ WASHINGTON STATE UNIVERSITY \\ UNIVERSITY OF WASHINGTON \\ AMERICAN MATHEMATICAL SOCIETY \\ CHEVRON RESEARCH CORPORATION \\ TRW SYSTEMS \\ NAVAL ORDNANCE TEST STATION
}




\section{Pacific Journal of Mathematics}

\section{Vol. 23, No. 1 \\ March, 1967}

M. J. C. Baker, A spherical Helly-type theorem ................... 1

Robert Morgan Brooks, On locally m-convex*-algebras.............. 5

Lindsay Nathan Childs and Frank Rimi DeMeyer, On automorphisms of separable algebras ...................................

Charles L. Fefferman, A Radon-Nikodym theorem for finitely additive set

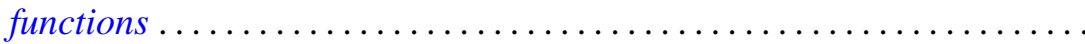

Magnus Giertz, On generalized elements with respect to linear

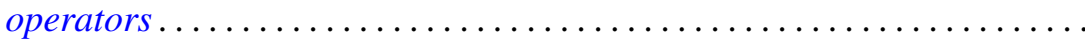

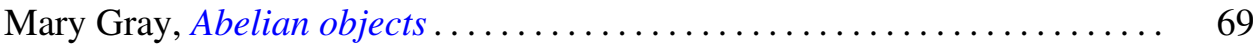

Mary Gray, Radical subcategories.............................. 79

John A. Hildebrant, On uniquely divisible semigroups on the two-cell . . . . . 91

Barry E. Johnson, AW*-algebras are $\mathrm{QW}^{*}$-algebras ............... 97

Carl W. Kohls, Decomposition spectra of rings of continuous functions . . . . 101

Calvin T. Long, Addition theorems for sets of integers .............. 107

Ralph David McWilliams, On $w^{*}$-sequential convergence and quasi-reflexivity ................................... 113

Alfred Richard Mitchell and Roger W. Mitchell, Disjoint basic

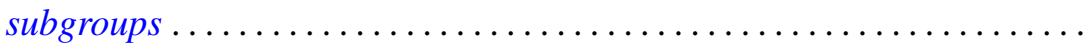

John Emanuel de Pillis, Linear transformations which preserve hermitian and positive semidefinite operators .

Qazi Ibadur Rahman and Q. G. Mohammad, Remarks on Schwarz's lemma

Neal Jules Rothman, An $L^{1}$ algebra for certain locally compact topological semigroups ...

F. Dennis Sentilles, Kernel representations of operators and their adjoints ...

D. R. Smart, Fixed points in a class of sets

K. Srinivasacharyulu, Topology of some Kähler manifolds

Francis C.Y. Tang, On uniqueness of generalized direct decompositions .

171 Albert Chapman Vosburg, On the relationship between Hausdorff dimension and metric dimension . 